their local areas to enable future access to shared care record systems as the benefits to hospices are considerable.

\section{P-101 IMPLEMENTING THE THERAPY OUTCOME MEASURE (TOM) IN A DAY HOSPICE}

Sally Boa, Gill Foster, Mandy Malcomson. Strathcarron Hospice, Denny, UK

\subsection{6/bmispcare-2019-HUKNC.124}

Background Hospice Day Services provide support to people to enable them to live with life limiting illness. The focus is on enhancing well-being and supporting people to continue to participate in activities that are important to them. Commonly used palliative care outcome measures tend to focus on problems and symptoms rather than well-being, activity and participation. The Therapy Outcome Measure (TOM) has been developed using the World Health Organization's International Classification of Functioning, Disability and Health and allows professionals to rate the person against descriptors which relate to four different domains: Impairment, Activity, Participation and Well-being.

Aim We aimed to find out if the TOM Palliative Care Scale could be used reliably and on a routine basis in Day Hospice by the multidisciplinary team to measure the impact of their interventions.

Methods Staff working in Day Hospice received training to become familiar with the TOM scales and use the scales reliably.

TOM was used with every patient referred to Day Hospice at two points during their episode of care; within the first two weeks and 12 weeks later. Patients were also assessed using the Australian Karnofsky Performance Status (AKPS), which provided information about functional status.

Findings Over a 22 week period, initial TOMs were used with 70 patients. Thirteen patients had TOM scores at 1 and 12 weeks as many patients deteriorated or stopped attending before the 12 week review. Changes in TOMs scores at these time points showed improvements in patients' well-being, activity and participation whilst impairment stayed the same. No changes were seen in AKPS level for this group.

Conclusions TOM scoring was readily adopted by those who received training. It was relatively quick to complete and provided meaningful information that reflected the impact of the interventions. Reducing the length of time between scores may enable collection of comparative scores for more patients.

\section{P-102 USING OUTCOME ASSESSMENT AND COMPLEXITY COLLABORATIVE MEASURES TO REDESIGN HOSPICE COMMUNITY TEAM}

Oliver-Jon Tidball, Christina Eldridge. Heart of Kent Hospice, Maidstone, UK

\subsection{6/bmispcare-2019-HUKNC.125}

Previous team composed of static level of Clinical Nurse Specialists (CNSs), increasing caseloads, limited prioritisation, silo working, resistance to change/new opportunities, and static budget. This led to high demand and high pressures with a rigid workforce. The need for change was identified to make the service fit for the present and incorporate future-proofing.

OACC was identified and developed as a means to achieve a skill mix team: right person, right place, right time, to improve caseload management. The existing team were put at risk of redundancy and given the opportunity to apply for a position with the new structure.

March 2018 - the majority of the existing team decided to take redundancy reducing the Hospice Community Team (HCT) to a few remaining team members along with some agency and bank staff during the recruitment phase. HCT were not fully staffed until October/November 2018 with geographical 'zones' and 'urgent response' teams introduced in December 2018.

Three OACC tools (IPOS, AKPS, Phase of Illness) are being used with Views On Care due to be introduced shortly.

\section{OACC impacts:}

- Re-design of the hospice referral form;

- Using OACC as part of admissions and discharges from IPU and clinical assessment process;

- Development of Clinical Drop-In Day;

- New MDT process focussed around 'unstable' and inactivating;

- Prioritisation of assessments in zones between Registered Nurses and Clinical Nurse Specialists/Advance Nurse Practitioners;

- Utilisation of Paramedic role in 'urgent response' team to assess unstable/crisis situations;

- HCA role to manage more 'stable' patients and support carers at end of life;

- Good communication essential between roles, zones and urgent response teams.

\begin{tabular}{lcc} 
Abstract P-102 Table 1 & & \\
\hline & February & February \\
& 2018 & 2019 \\
\hline Improved caseload management (total caseload) & 392 & 367 \\
Improved caseload management (average duration on & 382 days & 274 days \\
caseload) & & \\
Improved patient management (face to face assessments) & 233 & 291 \\
Improved speed of response (referral to 1st assessment) & 6 days & 4.5 days \\
\hline
\end{tabular}

\section{P-103 IMPLEMENTING THE INTEGRATED PALLIATIVE OUTCOME SCALE (IPOS) IN A SPECIALIST PALLIATIVE CARE SERVICE}

Mary Holloway, Victoria Barrett. Northamptonshire Healthcare NHS Foundation Trust, Northampton, UK

\subsection{6/bmispcare-2019-HUKNC.126}

Background Evidence shows that routine measurement of patient-reported outcomes (PROMs) is important in palliative care (Bausewein, Daveson, Currow, Downing et al., 2016; Collins, Witt, Bausewein, Daveson et al., 2015). Increasing emphasis is placed on PROMs by those who commission services (Bausewein, Schildmann, Rosenbruch, Haberland et al., 2018).

IPOS is a patient-reported outcome questionnaire in a suite of six palliative care-specific measures developed by the Outcome Assessment and Complexity Collaborative (OACC). OACC recommend a phased implementation (Witt, de WolfLinder, Dawkins, Daveson et al., 2015; Dawkins \& de WolfLinder, 2015). 
Aim Embed three measures from the OACC suite into clinical practice in our service.

Method Phased implementation began with identification of IPOS champions for each service area. Champions received training on Phase of Illness (POI) and Australia-Modified Karnofsky Performance Status (AKPS). Email communication was sent to all staff regarding OACC. Posters were displayed and project team members were available to answer questions. POI and AKPS were then routinely recorded across the service.

A training package was devised and delivered to all staff on using IPOS with patients. Community teams implemented IPOS into clinical practice first, followed by the inpatient units.

Initial audit used a randomised purposive sampling approach to obtain a sample of $10 \%$ of the caseload over the initial three month period which included patients from all service areas. The patient records for these patients was then reviewed using a standardised proforma to determine whether IPOS had been considered and completed where appropriate.

Results $84 \%$ of patients had at least one IPOS considered. IPOS was not considered at only $36 \%$ of encounters.

IPOS is now completed weekly and reviewed in MDT for all inpatients. It is considered at each encounter for community patients. Responses are used to guide clinical decisionmaking.

Conclusions Appropriate use of IPOS was much higher in our implementation period than reported for other services (Bausewein, Schildmann, Rosenbruch, Haberland et al., 2018).

We attribute this success to the quality and structure of staff training, phased implementation and to the visibility of the project team throughout the implementation process (Antunes, Harding \& Higginson on behalf of EUROIMPACT, 2014).

\section{P-104 THE EVOLUTION OF IMPACT REPORTING IN A HOSPICE DAY SERVICE}

Katherine Rugen, Aimee Doyle, Kate Dreyer, Chris Crompton, Kate McIntegart, Kate Marley. Woodlands Hospice, Liverpool, UK

\subsection{6/bmjspcare-2019-HUKNC.127}

Existing quantitative evidence for the benefits of Hospice Day Services is limited. Clinical Commissioning Group funding decisions are often based on numbers of attendees, rather than overall impact on individuals engaging with those services.

At Woodlands Hospice, an outcome measures questionnaire, the Integrated Palliative Care Outcome Scale (iPOS), was implemented on the inpatient unit several years ago, and, in addition to being clinically useful for individual patients, audit data showed an overall improvement in average iPOS score during patients' admission.

The use of iPOS was piloted for new patients to our Day Services at their initial assessment from December 2018. This tool was used to assist in transforming our individual patient care plan, with a change in our practice being to repeat iPOS for each patient every four weeks to update the care plan. A baseline audit showed a mean overall improvement in patients' iPOS score when repeated after four weeks. Interventions during the four-week intervening period varied according to the patient's own personalised plan of care. The interventions ranged from solely medical outpatient review, to multidisciplinary assessment and management, with some patients attending individual outpatient sessions only, and others attending group therapy sessions.

This audit looks at the breakdown in improvement of physical symptoms and psychological wellbeing, and compares the reduction in iPOS score to changes in other outcome measures, namely the OACC Phase of illness and the Palliative Performance Scale. The use of these outcome measures will enable the hospice to monitor the effect of the care and interventions it provides to each individual patient and ensure their personal care plan is continually adapted in accordance with their specific needs as their condition changes.

\section{P-105 MANAGEMENT OF A TRAINEE-LED RESEARCH COLLABORATIVE}

${ }^{1}$ Heena Khiroya, ${ }^{1}$ Natasha Freeman, ${ }^{1}$ Hazel Coop, ${ }^{1,2}$ Clare Marlow, ${ }^{1,3,4}$ Derek Willis. ${ }^{1}$ WM CARES, Wolverhampton, UK; ${ }^{2}$ New Cross Hospital, The Royal Wolverhampton NHS Trust, Wolverhampton, UK; ${ }^{3}$ Severn Hospice, Telford, UK; ${ }^{4}$ University of Chester, Chester, UK

10.1136/bmjspcare-2019-HUKNC.128

Background The West Midlands Collaboration Actioning Research in End of life and Supportive Care (WM CARES) is a palliative medicine trainee-led research collaborative, established in 2016. WM CARES enables trainees to be involved in research to benefit patient care and to gain experience in research prior to becoming consultants (Coop, Macfarlane, Twigger, Doré et al., 2017).

Published literature reveals several experiences of setting up trainee-led research collaboratives (Chamberlain, Schofield, Hancock, Etkind et al., 2019; Dowswell, Bartlett, Futaba, Whisker et al., 2014) but there is a paucity of information relating to the ongoing management and output of these collaboratives. Existing published work relates mainly to surgical specialties (Dowswell, Bartlett, Futaba, Whisker et al., 2014; Bhangu, Kolias, Pinkney, Hall et al.,2013; Jamjoom, Phan, Hutchinson \& Kolias, 2016).

Aim This work aims to describe our experience of managing and maintaining a trainee-led research collaborative within the field of palliative medicine. We wanted to understand potential barriers to maintaining a registrar-led research collaborative.

Methods We distributed a survey to the palliative medicine registrars in the West Midlands deanery in March 2019 to explore past, present and future levels of engagement with WM CARES.

Results Fourteen trainees (78\% of total) completed the survey. To date, WM CARES has published two papers (Coop \& Marlow, 2019; Macfarlane, Shayler, Nelms, Willis et al., 2018) and the latter has sparked the idea for the first project being conducted by the UK Palliative trainees Research Collaborative (Chamberlain, Schofield, Hancock, Etkind et al., 2019). Despite the pool of trainees changing since the conception of WM CARES, there is willingness to take on leadership roles within the collaborative, with all roles except one expected to be filled next year. The most common reasons cited for not taking on roles were maternity leave $(40 \%)$ and 